\title{
Immune therapy for treating type 1 diabetes: challenging existing paradigms
}

\author{
Jay S. Skyler \\ Diabetes Research Institute, University of Miami Miller School of Medicine, Miami, Florida, USA.
}

\begin{abstract}
Patients with type 1 diabetes (T1D) rapidly lose $\beta$ cell function and/or mass, leading to a life-long dependence on insulin therapy. $\beta$ Cell destruction is mediated by aberrant immune responses; therefore, immune modulation has potential to ameliorate disease. While immune intervention in animal models of diabetes has shown promising results, treatment of patients with T1D with the same agents has not been as successful. In this issue of the $J C I$, Haller and colleagues present data from a small clinical trial that tested the efficacy of a combination of immunomodulatory agents, anti-thymocyte globulin and pegylated granulocyte CSF, neither of which have shown benefit for T1D as single treatment agents. Many patients that received combination therapy maintained $\beta$ cell function at baseline levels up to a year after treatment. The results from this study challenge current trial design paradigm that for combined therapy to be successful individual agents should show benefit.
\end{abstract}

\section{The search for effective immune interventions for type 1 diabetes}

Type 1 diabetes (T1D) is considered to be an immune-mediated disease and thus potentially amenable to immune intervention (1). In animal models, a multitude of different immunologic approaches have been successful, including the use of single agents as well as a combination of agents selected for potential complementary effects (2). In patients with T1D, many immunologic strategies have been tested in phase II clinical trials; however, most of the results from these studies have been disappointing, with no single or combination treatment providing an unambiguous benefit in preserving $\beta$ cell function. In a few studies, individuals have shown transient benefit (3-6), while others have been without any observed benefit (7-10). Still other immune interventions have ambiguous effects that do not meet their primary outcome measure, such as preservation of $\beta$ cell function, but provide potential benefit based either on secondary outcome measures or mechanistic findings $(11,12)$. Some phase III clinical trials have also been conducted, though none of these treatments have shown benefit. The lack of success in these clinical trials has resulted in many researchers in the field taking a step back in order to assess the challenges and the opportunities for immune intervention in T1D and rethink the approaches to be used.

Interestingly, almost all of the clinical trials to date have enrolled subjects within the first 100 days after clinical diagnosis of T1D. It has been argued that the best responses are seen in patients who start immunotherapy as soon as possible after

Related Article: p. 448

Conflict of interest: The authors of the $J C I$ paper that is the subject of this Commentary are planning a follow-up study to be conducted within Type 1 Diabetes TrialNet, an NIH clinical trials network of which Jay S. Skyler is the chair. Jay S. Skyler was not involved in the study published in the $\mathrm{JCl}$. He reports ownership interest in Dance BioPharm, Dexcom Inc., Moerae Matrix Inc., Paean Therapeutics, SynAlpha Therapeutics, Tandem Diabetes Care, and VasoPrep Surgical. He has earned income from Boehringer Ingelheim, Halozyme Therapeutics, Intarcia Therapeutics, and Sanofi.

Reference information: / Clin Invest. 2015;125(1):94-96. doi:10.1172/JCI79190.

diagnosis of hyperglycemia; therefore, T1D ideally should be regarded as a "medical emergency," and immunologic treatment should be started within a few days of diagnosis (13).

A number of studies in animal models suggest that more robust results may be obtained by combining immunemodulating agents that have expected complementary mechanisms (14). Only a few clinical studies to evaluate the use of combination therapyhave been conducted, all of which failed to show benefit (15-17). One of the reasons that studies with combination therapy are lacking is that a demonstration that component therapies used together are safe is a requirement of regulating agencies. Moreover, for combination therapy, regulatory authorities ideally want each of the agents in the combined treatment to demonstrate individual efficacy. The few combination trials that have been allowed to go forward have used agents that have been used previously in combination in transplantation or some other setting.

\section{A paradigm-shifting combination}

In this issue, a study by Haller et al. examines the effects of a combination of lowdose anti-thymocyte globulin (ATG) and pegylated granulocyte CSF (G-CSF) on $\beta$ cell function in patients with T1D (18). This combination has previously shown beneficial effect in preclinical diabetes models (19). Although the study by Haller et al. is a very small study (only 25 subjects, randomized 2:1), it is important for several reasons. First, compared to typical studies, which enroll patients with a diabetes duration of less than 3 months, Haller and colleagues enrolled subjects with a diabetes duration ranging from 4 to 24 months, thus challenging the "medical emergency" paradigm. Indeed, at ClinicalTrials.gov, the Haller et al. study was titled "Reversing Type 1 Diabetes After It Is Established" (Clinicaltrials.gov 
NCT01106157). Second, after 12 months of follow-up, $\beta$ cell function was actually retained at approximately baseline levels in 9 of 16 subjects in the treated group. This observation is important, because rather than showing a difference between the experimentally treated subjects and placebo-treated subjects, as has been the case in most previous studies, these results suggest maintenance of $\beta$ cell function as a new paradigm for desired treatment effect. Third, the beneficial effect at 12 months was seen in response to a treatment that involved only 2 days of ATG administration and 12 weeks of G-CSF, suggesting that at least relative immune tolerance may have been achieved. Fourth, although there were a number of side effects seen in the experimental group, including cytokine release syndrome and serum sickness, it should be noted that these side effects are transient syndromes that appeared only at the time of drug exposure and are fully reversible, and, therefore, they should be categorized as intolerabilities rather than major adverse effects. Fifth, to the best of our knowledge, this is the first study to demonstrate preservation of $\beta$ cell function in T1D using a combination of agents. Sixth, Haller and colleagues used ATG and G-CSF, neither of which preserve $\beta$ cell function when used alone $(9,20)$, thus challenging the regulatory paradigm that agents used in combination should first show some individual benefit.

It should be noted that the sample size was very small and that there were some baseline differences between individuals in the experimental and placebo groups. These caveats preclude the ability to make unambiguous conclusions from this pilot study. A larger clinical study on the efficacy of ATG and G-CSF is expected to commence soon (Clinicaltrials.gov NCT02215200). If this larger trial produces similar results, this combination therapy will be a major advance in the treatment of T1D. The planned study, however, will differ from the study by Haller and colleagues, in that it will be enrolling subjects in the more conventional 100-day after diagnosis window.

\section{Future questions for combined therapy trials}

The beneficial effect of combined therapy with agents that individually are not effec- tive raises questions as to how future combination therapy trials should be designed and how to determine the optimal types of agents to try in combination. Treatments that combine more than two agents should also be considered, as multiple pathways are involved in the etiopathogenesis of T1D, including inflammation and innate immunity, classical adaptive immunity, and a vulnerable $\beta$ cell. A potential strategy moving forward might involve a combination of the following: an antiinflammatory agent that targets the innate immune system; one (or more) agents that target the adaptive immune system; antigen-specific immunomodulatory agents, such as oral or nasal insulin, glutamic acid decarboxylase vaccine, and/or proinsulin peptides (all of which have demonstrated beneficial effects in animal models and safety in patients); Treg-promoting agents; and agents such as GLP-1 receptor agonists that preserve $\beta$ cell health and improve $\beta$ cell function. While such a multifactorial approach may seem ambitious and complicated, the use of different agents does not need to be simultaneous, but they could be given sequentially. Moreover, the only agents in the above list with potentially serious, unquantified risks are those that target the adaptive immune system. This is not to say that a multicomponent approach is without risk or that regulatory agencies, ethics committees, and potential research subjects will readily accept the concept of combined therapy. On the other hand, if the larger planned trial confirms the results of Haller and colleagues that a combination of ATG and G-CSF maintains $\beta$ cell function, the next step will be to test other combinations of immunomodulatory agents. The goal of such a combined approach is to find a treatment that actually increases $\beta$ cell function, potentially obviating the need for insulin therapy for patients with T1D. While such a strategy is not feasible at present, if we proceed with caution, the winning combination might be found. The first paradigms have been challenged by Haller and colleagues, it is time to challenge more.

Address correspondence to: Jay S. Skyler, Diabetes Research Institute, 1450 NW 10 Ave. - Suite 3054, Miami, Florida 33136, USA. Phone: 305.243.6146; E-mail: JSkyler@med.miami.edu.
1. Eisenbarth GS. Banting Lecture 2009: an unfinished journey: molecular pathogenesis to prevention of type $1 \mathrm{~A}$ diabetes. Diabetes. 2010;59(4):759-774.

2. Shoda LK, et al. A comprehensive review of interventions in the NOD mouse and implications for translation. Immunity. 2005;23(2):115-126.

3. Herold KC, et al. A single course of anti-CD3 monoclonal antibody hOKT3gamma1(Ala-Ala) results in improvement in C-peptide responses and clinical parameters for at least 2 years after onset of type 1 diabetes. Diabetes. 2005;54(6):1763-1769.

4. Keymeulen B, et al. Four-year metabolic outcome of a randomised controlled CD3-antibody trial in recent-onset type 1 diabetic patients depends on their age and baseline residual beta cell mass. Diabetologia. 2010;53(4):614-623.

5. Pescovitz MD, et al. B-lymphocyte depletion with rituximab and $\beta$-cell function: two-year results. Diabetes Care. 2014;37(2):453-459.

6. Orban T, et al. Costimulation modulation with abatacept in patients with recent-onset type 1 diabetes: follow-up 1 year after cessation of treatment. Diabetes Care. 2014;37(4):1069-1075.

7. Wherrett DK, et al. Antigen-based therapy with glutamic acid decarboxylase (GAD) vaccine in patients with recent-onset type 1 diabetes: a randomised double-blind trial. Lancet. 2011;378(9788):319-327.

8. Moran A, et al. Interleukin-1 antagonism in type 1 diabetes of recent onset: two multicentre, randomised, double-blind, placebo-controlled trials. Lancet. 2013;381(9881):1905-1915.

9. Gitelman SE, et al. Antithymocyte globulin therapy for patients with recent-onset type 1 diabetes: a randomized double-blind phase 2 trial. Lancet Diabetes Endocrinol. 2013;1(4):306-316.

10. Knip M, et al. Hydrolyzed infant formula and early $\beta$-cell autoimmunity: a randomized clinical trial. JAMA. 2014;311(22):2279-2287.

11. Skyler JS, et al. Effects of oral insulin in relatives of patients with type 1 diabetes: The Diabetes Prevention Trial - Type 1. Diabetes Care. 2005;28(5):1068-1076.

12. Rigby MR, et al. Targeting of memory T cells with alefacept in new-onset type 1 diabetes (T1DAL study): 12 month results of a randomised, doubleblind, placebo-controlled phase 2 trial. Lancet Diabetes Endocrinol. 2013;1(4):284-294.

13. Bach JF. Anti-CD3 antibodies for type 1 diabetes: beyond expectations. Lancet. 2011;378(9790):459-460.

14. Matthews JB, et al. Developing combination immunotherapies for type 1 diabetes: recommendations from the ITN-JDRF Type 1 Diabetes Combination Therapy Assessment Group. Clin Exp Immunol. 2010;160(2):176-184.

15. Gottlieb PA, et al. Failure to preserve $\beta$-cell function with mycophenolate mofetil and daclizumab combined therapy in patients with new- onset type 1 diabetes. Diabetes Care. 2010;33(4):826-832.

16. Long SA, et al. Rapamycin/IL-2 combination therapy in patients with type 1 diabetes augments Tregs yet transiently impairs $\beta$-cell function. Diabetes. 2012;61(9):2340-2348.

17. Griffin KJ, Thompson PA, Gottschalk M, Kyllo 
JH, Rabinovitch A. Combination therapy with sitagliptin and lansoprazole in patients with recent-onset type 1 diabetes (REPAIR-T1D): 12-month results of a multicentre, randomised, placebo-controlled, phase 2 trial. Lancet Diabetes Endocrinol. 2014;2(9):710-718.
18. Haller MJ, et al. Anti-thymocyte globulin/G-CSF treatment preserves $\beta$ cell function in patients with established type 1 diabetes. JClin Invest. 2015;125(1):448-455.

19. Parker MJ, et al. Immune depletion with cellular mobilization imparts immunoregulation and reverses autoimmune diabetes in nonobese diabetic mice. Diabetes. 2009;58(10):2277-2284.

20. Haller M, et al. Granulocyte colony stimulating factor (GCSF) fails to preserve $\beta$-cell function in patients with recent onset type 1 diabetes (T1D) Diabetes. 2014;63:A428. 